\title{
A Comparison between Natural and Synthetic Food Flavoring Extracts Using Infrared Spectra and Optical Activity
}

\author{
Parekhan Aljaff ${ }^{1}$, Banaz O.Rasheed ${ }^{2}$ and Trifa Attar Omer ${ }^{3}$
}

\begin{abstract}
Food is the basic necessity of life. One works hard and earns to satisfy our hunger. But at the end of the day, many of us are not sure of what we eat. We may be eating a dangerous flavors and dyes. Often, we invite diseases rather than good health. The purpose of this article is to detect the presence of food adulterants in some common foods and to create awareness about the artificial tests and dyes. A study of the IR spectra and the optical activitiy of two natural and artificial most common used flavor and colors (Vanilla and Strawberry) were detected. IR spectra of synthetic Vanilla were dominated by specific peaks that attributed to corresponding synthetic pigments (specific spectral band of stretching $C=0$ ester of aldehydic and ketonic groups in synthetic flavor at 1744.87 $\mathrm{cm}^{-1}$ with a weak shoulder at $1700 \mathrm{~cm}^{-1}$. And stretching CO of sucrose at $(990.49$ and 923,70$)$ $\mathrm{cm}^{-1}$. The synthetic Strawberry characterized with specific spectral bands of $\left(C=O\right.$ stretching at $1634.96 \mathrm{~cm}^{-1}$ in ester and CO stretching of sucrose at $925 \mathrm{~cm}^{-1}$ ), while these functional groups disappeared in natural Vanilla and Strawberry extracts. The natural Flavoring extracts posse's levorotatory property; they are optically active, while the synthetic extracts not rotates the plane of polarization of the light which passes through the material, they are said to be; not active optically.

The obtained results indicated that, Infrared spectrum and Optical activity could be adapted to detect adulterants added products, and to differentiate between natural and artificial food flavoring extracts.

Key Words: Adulteration, Food dyes, Flavors, Vanilla, Strawberry.
\end{abstract}

\section{Introduction}

Adulterant means any material which is or could be employed for making the food unsafe or substandard or misbranded or containing extraneous matter; Food is adulterated if its quality is lowered or affected by the addition of substances which are injurious to health or by removal of substances which are nutritious. Some of the common additive foods are Colors and Flavors. [1].The adulteration are frequently involves there replacement of high cost ingredients with cheaper substitutes. Drinks subjected to be adulterated with other synthetic flavors and colorants of lower commercial value. Although the adulteration is done for economic reasons, the action can affect the chemical composition and quality parameters of food.

Artificial food dyes and testes are one of the widest spread and critically important groups of Pollutants [2]. A single artificial flavoring can be a combination of hundreds of individual chemicals, "Prior to the 1950 's, the coloring of the nation's food supply was a simple process. If manufacturers wanted to add color to a food or candy it was done primarily with natural plant and vegetable based compounds. Now food adulteration is an act of internationally debasing the quality of food offered for sale by admixture of inferior. Most artificial food dyes and testes are made from petroleum oil and coal tar. Manufacturers use color additives to cover up an absence of natural color.

Adulteration of food can pose serious risk to health in some cases $[3,6]$. Hyper kinesis and Learning Disabilities (H, LD) Linked to the ingestion of Artificial Food Colors and Flavors [2].

In 1997, a research was conducted at the University of south Florida; exposure of mice to a mixture of 5 dyes including blue, green, red, yellow and orange. (This more approximates what a child would receive in the real world on a daily basis).The food dyes were given to the rat pups beginning at 5 days of age continuing throughout the first month of postnatal life. Amounts given were not in excess, but were calculated to equal the average daily intake for American children ( $1 \mathrm{mg} / \mathrm{kg}$ body weight).

After exposure to food dyes, there was enough evidence for the researchers to conclude that the Learning ability, (in this case called "avoidance learning"), was weakened in test animals exposed to the food colors, "food dyes affect activity levels during the first month of postnatal life." And there was an increase in activity. This supports the assumption that lowered brain dopamine levels increase hyperactivity. There conclusion was that, petroleum based chemicals in food (artificial flavors and colors) can harm child behavior [4].

In 2006, almost 19 million pounds of color additives were certified by FDA inspectors. The FDA receives a "user fee" from the manufacturer for each pound of food dye certified. That means each pound approved, not each pound examine [5]. In commercially available FD\&C Yellow \#5 and \#6,benzidine (which causes cancer) has been found in amounts up to 200 times the officially allowed level of only 1 part per billion[3]. 


\section{A- Vanilla flavorings}
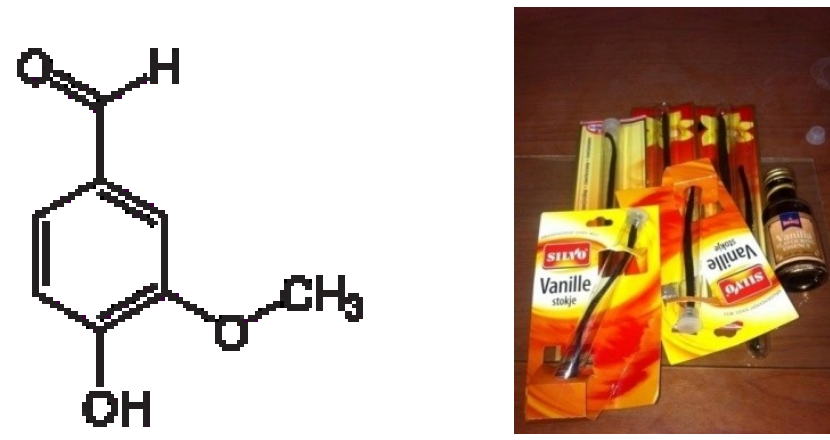

The molecular formula for vanillin is $\mathrm{C}_{8} \mathrm{H}_{8} \mathrm{O}_{3}$. Vanilla extract is widely used as a flavoring ingredient in foods beverages, cosmetic, pharmaceutical and tobacco industries. It contains approximately 200 substances. The main compounds are vanillin, 4-hydroxybenzaldehyde, vanillin acid and 4-hydroxybenzoic acid. Since natural vanilla extract is limited and prices are high, artificial vanilla flavorings are often used. Due to quality and price concerns, it is important to differentiate between these two types.

There are two types of artificial vanilla flavorings, which always contain vanillin that is synthesized from cheap raw material such as guaiacol, eugenol or lignin, a natural polymer found in wood; and/or ethyl vanillin is added, which is another artificially produced vanilla compound that has three times the flavor strength of vanillin [6,7].

Most countries have regulations to control the content of synthetic vanilla products and by-products such as the US Code of Federal Regulations from1988, which requires that beverage alcohol products are labeled, if synthetic flavors are used [8]

An average sized person would probably need to eat over 75 grams (2.5 ounces) of pure vanillin for it to have a toxic effect. The established "safe" daily intake of vanilla is $10 \mathrm{mg}$ per $\mathrm{kg}$.

The production of vanilla beans is quite expensive, since it is a very labor intensive process and harvesting takes place two to three years after planting [9]. There is no difference between the chemistry of natural and artificial food flavoring, adverse health effects are believed to come from the artificial flavoring [10].

\section{B. Strawberry as food test and color}

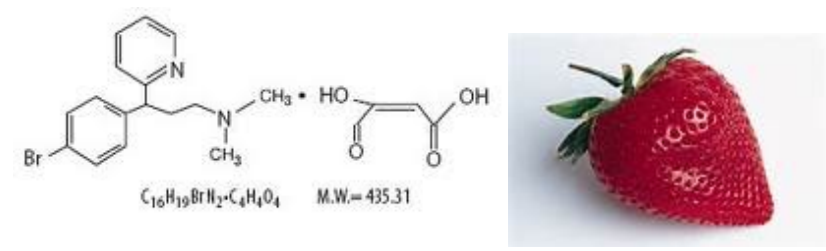

In USA Kellogg's Strawberry Nutrigrain bars are colored with Red 40, Yellow 6 and Blue 1 [11]. The most widely used dyes are Red 40.They contain carcinogens and may cause allergic reactions. Each year, about 15 million pounds of synthetic dyes go into the U.S. food supply (well, the "processed" U.S. food supply).

Since 1955 the consumption of food color additives has increased by five times, the U.S. eats a lot of processed foods.

There are some potential problems with synthetic food dyes, specifically:

Carcinogenicity - leading to cancer development

Genotoxicity - leading to mutations or damaging chromosomes

Neurotoxicity - leading to the damage of nerve tissues [12].

\section{How do natural molecules exhibit Optical Rotation?}

A monochromatic linearly polarized light beam can be considered as a superposition of two circularly polarized electromagnetic waves that are propagating in the same direction with the same frequency but the opposite sense of rotation. The plane of polarization of the resulting linearly polarized wave thus prepared can be changed (rotated) by applying a phase shift between its two circularly polarized components. With the help of this concept we can explain the phenomenon of optical rotation. The chiral material interacts slightly differently with the two circularly polarized components of a linearly polarized light beam. This is true both for absorption and refraction. A chiral medium have slightly different refractive indices for Left- and right hand circularly polarized light beams This means that even if they are not absorbed they travel at different speeds 
through the medium. Therefore, this causes a phase shift between the two circularly polarized components which increases proportional to the path length that the light travels through the chiral medium. This phase shift manifests itself as a rotation of the plane of polarization of the resultant linearly polarized light beam.

Sample that contains only one enantiomer of achiral molecule is said to be optically pure. The enantiomer that rotates light to the right, or clockwise when viewing in the direction of light propagation, is called the dextrorotatory (d)or $(+)$ enantiomer, and the enantiomer that rotates light to the left, or counterclockwise, is called the levorotatory or (-) enantiomer.

\section{Materials And Methods:}

Natural Vanillawas purchased from Sweden market because it is not available in our local markets. Fresh Strawberry purchased from Sulaimani local market.

Vanilla and Strawberry extract were prepared in chemistry department of college of Science, Faculty of Science and science Education, University of Sulaimani .

Artificial, Vanillin and artificial Strawberry tests purchased from a local supermarket, with cheap price are produced in India for Natco Foods Ltd.

Infrared spectra obtained using FT-IR LR 64912C Spectrometer/Perkin Elmer UK. The optical activity of the compounds obtained using ATAGO POLAX-2L (atago.co.LTD-Japan) Polarimeter with polarized sodium light.

\section{3a. Preparation of Vanilla extract}

\section{Experimental Part}

Vanilla extracted by dissolving extract of $(1 \mathrm{mg})$ of Vanilla beans in $10 \mathrm{ml}$ of $45 \%$ by volume ethanol, after evaporation of the solvent natural Vanilla oil obtained, Vanilla is strong polar, with sweet test because it is bounded chemically with sugar (glucose).

\section{3b.Strawberry extract preparation}

Strawberry Extracted by dissolving 5mg of Strawberry in $50 \mathrm{ml}$ of absolute ethanol for 46 hour, the solvent evaporated to obtain Strawberry oil.

Strawberry additive is also strong polar, acidic in test because it is chemically bonded with acid.

The natural and the artificial Strawberry were purchased from our local market

\section{3c.The infrared spectrum}

IR spectrum of the prepared and the synthetics Vanilla and Strawberry has been detected and the main corresponding function group assignment of all extracted food additives are determined and shown in the following figures and tables.

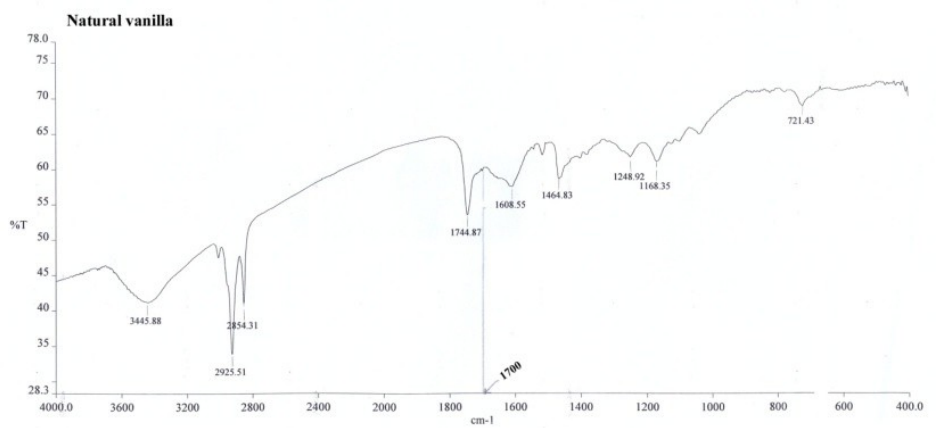

Figure (1) Infrared Spectrum of natural vanilla

Table (1) Vibration assignment of natural Vanilla

\begin{tabular}{|c|c|}
\hline$v$, Observed $\left(\mathrm{cm}^{-1}\right)$ & Function group assignment \\
\hline 3459.32 & $\begin{array}{l}\text { More possible: } O \text {-H stretching of alcohol (broad band) and } \\
\text { may be of phenol. }\end{array}$ \\
\hline $2972.48 ; 2870$ & $\mathrm{C}$-H stretching of $-\mathrm{CH}_{3}$ and $-\mathrm{CH}_{2}$ groups. \\
\hline 2820 & As weak shoulder C-H stretching of aldehydic hydrogen. \\
\hline 1670 & $\begin{array}{l}\text { Shoulder nearly at } \\
1670 \mathrm{~cm}^{-1} \text { more possible of } C=O \text { stretching of aromatic } \\
\text { aldehyde. }\end{array}$ \\
\hline 1670 & More possible $C=O$ stretching of aromatic aldehyde. \\
\hline 1652.92 & $\begin{array}{l}\mathrm{C}=\mathrm{C} \text { stretching of aromatic ring in mesomeric form due to } \mathrm{O} \text { - } \\
\mathrm{CH}_{3} \text { group. }\end{array}$ \\
\hline
\end{tabular}




\begin{tabular}{|l|l|}
\hline 1455.81 & C-H bending of CH2 group. \\
\hline 1410 & O-H bending of alcohol. \\
\hline $1135.63: 1046.76$ & More possible of C-O alcohol or ether. \\
\hline $990.49 ; 923.76$ & stretching $C O$ of sucrose \\
\hline
\end{tabular}

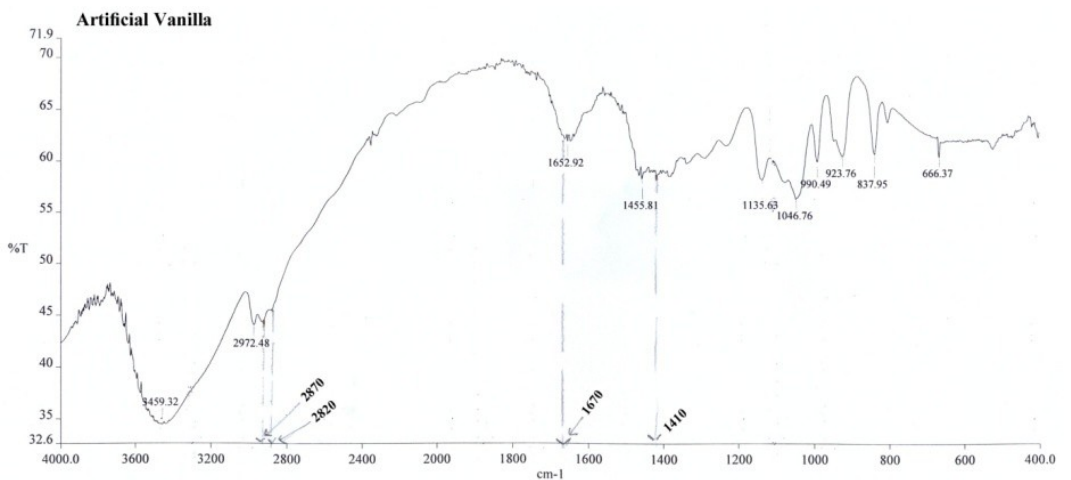

Figure (2) Infrared Spectrum of Artificial vanilla.

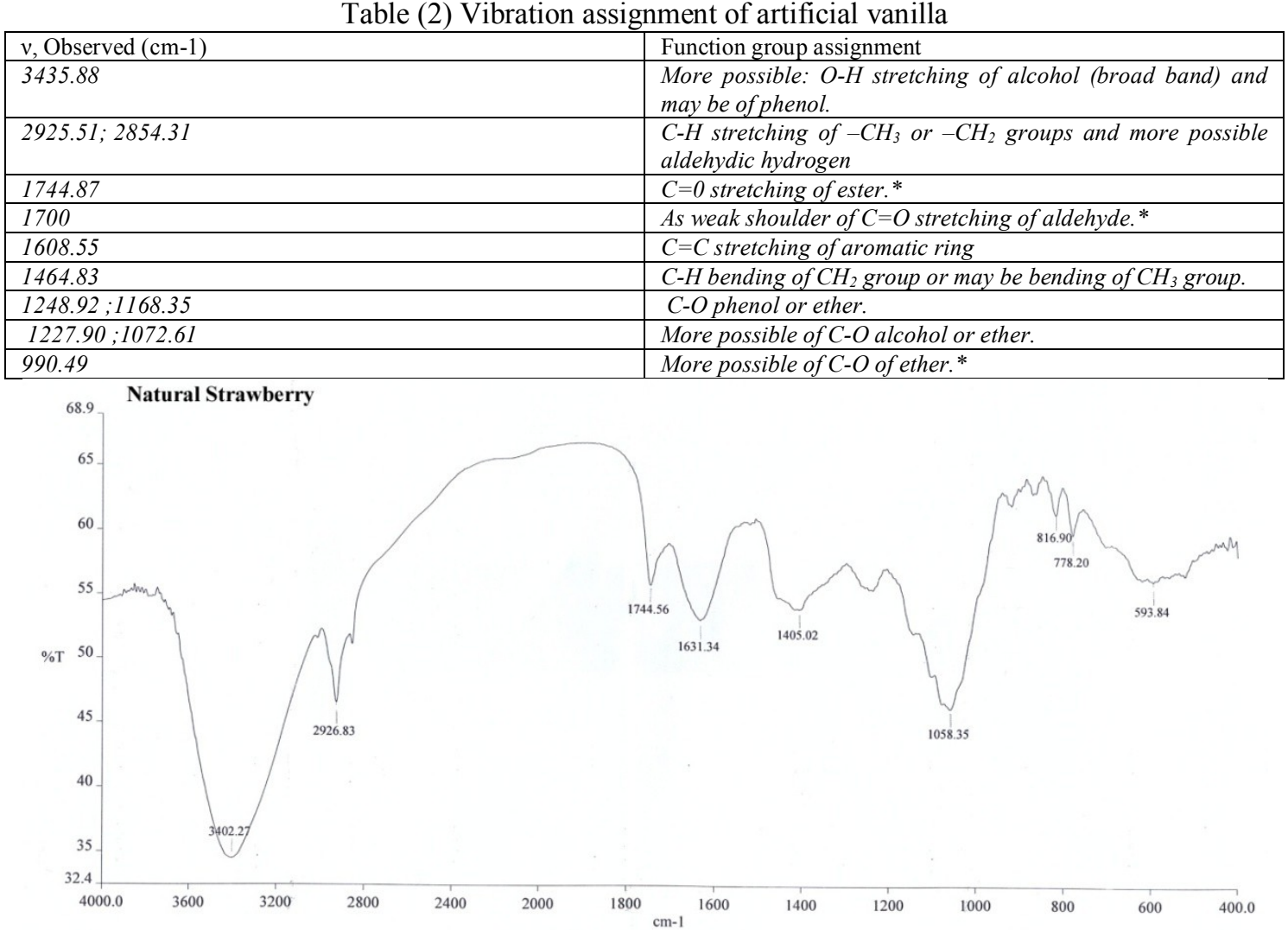

Figure (3) Infrared Spectrum of natural Strawberry

Table (3) Vibration Assignment of natural Strawberry

\begin{tabular}{|l|l|}
\hline$v$, Observed $\left(\mathrm{cm}^{-1}\right)$ & Function group assignment \\
\hline 3402.27 & $\begin{array}{l}\text { More possible: } \text { - } H \text { stretching of alcohol (broad band) and } \\
\text { may be of phenol. }\end{array}$ \\
\hline 2926.83 & $\begin{array}{l}\text {-H stretching of alkyl group } \\
\text { N-H bending of amine group and possible and may be salt of } \\
\text { secondary amine. }\end{array}$ \\
\hline 1405.02 & O-H bending of alcohol. \\
\hline 1058.35 & -O stretching of alcohol. \\
\hline $816.90 ; 778.20$ & - H bending of aromatic ring disubstituted (meta). \\
\hline 593.84 & - Cl stretching. \\
\hline
\end{tabular}




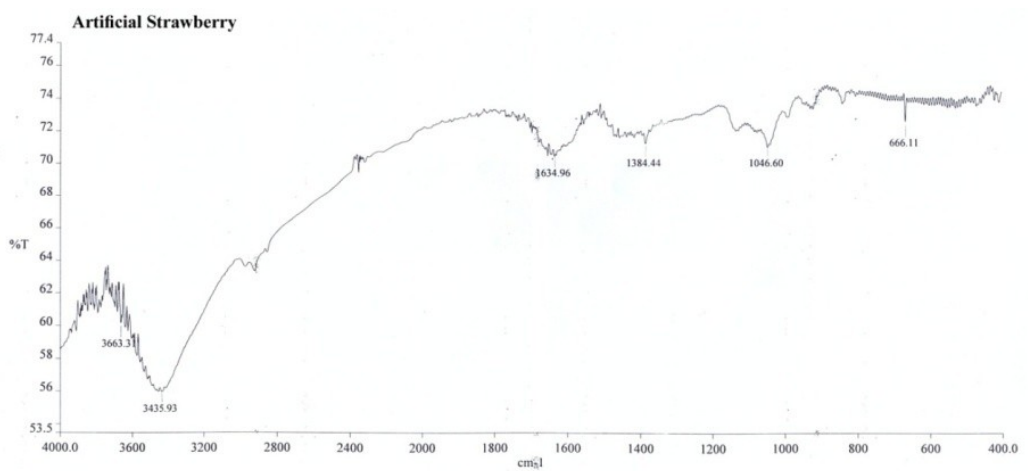

Figure (4) Infrared Spectrum of artificial Strawberry.

Table (4) Vibration assignment of artificial Strawberry.

\begin{tabular}{|l|l|}
\hline$v$, Observed $\left(\mathrm{cm}^{-1}\right)$ & Function group assignment \\
\hline 3663.31 & O-H stretching free or $N$-H free. \\
\hline 3435.03 & $\begin{array}{l}\text { O- } H \text { stretching bonded and possible of } N \text { - } H \text { stretching of } \\
\text { secondary amine or } N H 2\end{array}$ \\
\hline 1634.96 & $C=O$ stretching of ester* \\
\hline 1384.44 & $C$ - bending of alkyl group. \\
\hline 1046.60 & $C$-O stretching of ether or may be of $C$ - $N$ stretching of amine. \\
\hline 925 & stretching $C O$ of sucrose $*$ \\
\hline $816.90 ; 778.20$ & $C$ - bending of aromatic ring distributed (meta).. \\
\hline 666.11 & $C$-Cl stretching of aryl halide. \\
\hline
\end{tabular}

\section{3d.Optical activity detection:}

The polarimeter with sodium lamp detector used to study the optical activities of the studied food flavorings, the results are shown in table (5)

Table (5) Optical activity of the natural and artificial food additives

\begin{tabular}{|c|c|}
\hline Food additive & $\begin{array}{c}\text { Optical Rotation angle } \\
\text { (degree) }\end{array}$ \\
\hline Artificial Vanilla & 0.00 \\
\hline Natural Vanilla & Negative change in polarized light orientation value \\
\hline Artificial Strawberry & 0.00 \\
\hline Natural Strawberry & Negative change in polarized light orientation value \\
\hline
\end{tabular}

The polarimeter with sodium lamp detector shows that the orientation of the polarized light is zero for artificial food additives due to either these additives are uncontained chiral centers or as racemic modification. Contrary the natural food additives have changed the plane polarized light direction in the negative direction with certain value due to contained chiral centers.

- IR spectra shows the main corresponding function groups of all extracted food additives.

- Vanilla is an important flavor for many foods. Vanilla beans have been shown to contain over 200 compounds, which can vary in concentration depending on the region where the beans are harvested. Several compounds including vanillin, p-hydroxybenzaldehyde, guaiacol, and anise alcohol have been found to be important for the aroma profile of vanilla. Because of the complexity of the vanilla aroma profile there are many gaps in the current understanding of how vanilla compounds are volatilized in food systems.

\section{Results And Discussion:}

On based of our extractions of Natural food additives Vanilla and Strawberry the Vanilla shows strong polar and sweetness while the strawberry shows acidic taste due to their chemically bonding either with sugar (glucose) molecule or acid.

IR spectra show the main corresponding function groups of all extracted food additives.

Vanilla and Strawbery functional groups include aldehyde, ether and phenol.

From IR results we can conclude that, the synthetic tests can be characterized with specific bands of stretching $\mathrm{C}=\mathrm{O}$ ester of aldehydic and ketonic groups which used in the manufacturing process of synthetic tests at 1700 $1734 \mathrm{~cm}-1$ and stretching $\mathrm{CO}$ of sucrose at $996-963 \mathrm{~cm}-1$, while these functional groups disappeared in natural flavors. These functional groups are designated by a (*) in tables 2 and 4. IR spectra of synthetic Vanilla were dominated by specific peaks that attributed to corresponding synthetic pigments $\left(C=0\right.$ stretching at $1744.87 \mathrm{~cm}^{-1}$ 
in ester. a weak shoulder of $\mathrm{C}=\mathrm{O}$ stretching of aldehyde at $1700 \mathrm{~cm}^{-1}$ and $\mathrm{C}-\mathrm{O}$ of sucrose at $\left.990.49 \mathrm{~cm}^{-1}\right)$.The synthetic Strawberry characterized with specific spectral bands of $\left(\mathrm{C}=\mathrm{O}\right.$ stretching at $1734 \mathrm{~cm}^{-1}$ in ester , 925 $\mathrm{cm}^{-1} \mathrm{CO}$ stretching of sucrose), while these functional groups disappeared in natural Vanilla and Strawberry extracts.

The natural Flavoring extracts posse's levorotatory property; they are optically active, while the synthetic extracts not rotates the plane of polarization of the light which passes through the material.

While the natural Vanilla, and Strawberry are extracted from natural food additives they contained chiral centers, they, rotates direction of the polarized light to the left, or counterclockwise, they posse's levorotatory property; they are optically active.

As well the artificial additives are ionic or mesomeric molecules with rotational angle of zero degrees. This means that the artificial samples not contains an excess of one enantiomer of a chiral molecule, they are not active optically.

\section{References}

[1]. Quick Test For Some Adulterants In Food-Instruction Manual-part 1 .3/8/2012.

[2]. Ben F. Feingold, MD Journal of Learning Disabilities . VOIUME, 9, NUMBER,9 ,1976

[3]. K. Anderson, H. Kaplan, D. Lam and J. Lancaster (1999) Paternal Care by Genetic Fathers and Stepfathers II: Reports by Xhosa High School Students. Evolution and Human Behavior 20(6):433-452.

[4]. Richard W. Pressinger ( M.Ed.) s project supervised by Kofi Marfo at :The University of South Florida Special Education Department. 1997.

[5]. K. Anderson, H. Kaplan, and J. Lancaster (1999) Paternal Care by Genetic Fathers and Stepfathers I: Reports from Albuquerque Men. Evolution and Human Behavior 20(6):405-432., Food \& Drugs, Title 212

[6]. "Food additives and hyperactive behavior in 3-year-old and 8/9-year-old children in the community: a randomized, double-blinded, placebo-controlled trial", Lancet, Sept 2007

[7]. Herrmann A., Stockli M., J. Chrom., 1982, 246, 313-316.

[8]. 125. Vanillin (FAO Nutrition Meetings Report Series 44a).

[9]. Code Fed.Regulat. (1988) Title 21, Part189, sec. 189.130, Government Printing Office, Washington.

[10]. Rapid Differentiation Between Natural and Artificial Vanilla Flavorings for Determining Food Fraud Using AxION DSA/TOF with No Chromatography or Sample Preparation Published in USA, September 15, 2011Publication Number 5990-8716ENPerkinElmer, Inc. 940 Winter Street Waltham, MA 02451 USA .

[11]. U.S. Department of Labor, Occupational Safety \& Health Administration (OSHA).(2010). http://www.osha.gov/SLTC/flavoringlung/diacetyl_worker_alert.html.

[12]. Precisionnutition-All about Food Colour Additives. by Ryan Andrews | September27th, 2010.

[13]. Lucarelli MR, et al. Toxicity of food and drug cosmetic blue number 1 dye in critically ill patients. Chest 2004;125:793-795.

[14]. Ramasami, P., Jhaumeer-Laulloo, S., Rondeau, P., Cadet, F., Seepujak, H., \& Seeruttun, A. (2004). Quantification of sugars in soft drinks and fruit juices by density, refractometry, infrared spectroscopy and statistical methods. South African .Journal of Chemistry, $57,24-27$. 